Homology, Homotopy and Applications, vol.13(2), 2011, pp.19-36

\title{
ON THE VANISHING OF COHOMOLOGY IN TRIANGULATED CATEGORIES
}

\author{
PETTER ANDREAS BERGH
}

(communicated by J.P.C. Greenlees)

\begin{abstract}
We study the vanishing of cohomology in a triangulated category, in particular vanishing gaps and symmetry.
\end{abstract}

\section{Introduction}

In this paper, we study the vanishing of cohomology in a triangulated category $\mathcal{T}$ with suspension functor $\Sigma$. Given two objects $X$ and $Y$ of $\mathcal{T}$, a very natural question arises when looking at their cohomology: can we detect the vanishing of $\operatorname{Hom}_{\mathcal{T}}\left(X, \Sigma^{n} Y\right)$ for large $n$ by looking at finite vanishing gaps? That is, is there a finite set $S$ of integers such that the implication

$$
\operatorname{Hom}_{\mathcal{T}}\left(X, \Sigma^{n} Y\right)=0 \text { for } n \in S \Longrightarrow \operatorname{Hom}_{\mathcal{T}}\left(X, \Sigma^{n} Y\right)=0 \text { for } n \gg 0
$$

holds? The bounded derived category of a commutative local complete intersection ring is an example where this is true. Namely, for such a ring $A$, the following was shown by Jorgensen in [26] for a module $M$ of complexity $d$ : if $N$ is an $A$-module, and there exists an integer $n>\operatorname{dim} A$ such that $\operatorname{Ext}_{A}^{i}(M, N)=0$ for $n \leqslant i \leqslant n+d$, then $\operatorname{Ext}_{A}^{i}(M, N)$ vanishes for all $i>\operatorname{dim} A$. Since $\operatorname{Ext}_{A}^{n}(M, N)$ is isomorphic to $\operatorname{Hom}_{D^{b}(A)}\left(M, \Sigma^{n} N\right)$, the result of Jorgensen shows that we can indeed detect vanishing of cohomology in the bounded derived category $D^{b}(A)$ from finite vanishing gaps. Moreover, by a classical result of Gulliksen (cf. [23]), the complexity of an $A$-module is at most the codimension $c$ of $A$. Therefore, we can always detect vanishing of cohomology over $A$ by looking at gaps of length $c+1$.

Another natural question is: does symmetry hold in the vanishing of cohomology in $\mathcal{T}$ ? In other words, if $X$ and $Y$ are objects in $\mathcal{T}$ such that $\operatorname{Hom}_{\mathcal{T}}\left(X, \Sigma^{n} Y\right)$ vanishes for $n \gg 0$, then does it necessarily follow that $\operatorname{Hom}_{\mathcal{T}}\left(Y, \Sigma^{n} X\right)$ also vanishes for $n \gg 0$ ? Again, commutative local complete intersection rings provide examples where this holds. Namely, it was shown in [5] that if $M$ and $N$ are modules over such a ring $A$, then the implication

$$
\operatorname{Ext}_{A}^{i}(M, N)=0 \text { for } i \gg 0 \Longrightarrow \operatorname{Ext}_{A}^{i}(N, M)=0 \text { for } i \gg 0
$$

holds. Another class of rings where such symmetry holds are group algebras of finite

The author was supported by NFR Storforsk grant no. 167130 .

Received December 17, 2010, revised May 20, 2011; published on September 14, 2011.

2000 Mathematics Subject Classification: 16E30, 18E30, $18 \mathrm{G} 15$.

Key words and phrases: triangulated category, vanishing of cohomology.

Article available at http://intlpress.com/HHA/v13/n2/a2 and doi:10.4310/HHA.2011.v13.n2.a2

Copyright (C) 2011, International Press. Permission to copy for private use granted. 
groups (cf. [28]), or, more generally, as we shall see, symmetric algebras with "finitely generated" cohomology.

The two questions raised are studied in Section 3 and Section 4, respectively. We obtain affirmative answers when certain cohomology groups are finitely generated as modules over a ring acting centrally on our triangulated category, a concept we define in the following section. In particular, we show that Ext-symmetry holds for symmetric periodic algebras.

\section{Preliminaries}

Throughout this paper, we fix a triangulated category $\mathcal{T}$ with a suspension functor $\Sigma$. Thus $\mathcal{T}$ is an additive $\mathbb{Z}$-category together with an automorphism $\Sigma$ and a class of distinguished triangles satisfying Verdier's axioms (cf. [34]).

Recall that a thick subcategory of $\mathcal{T}$ is a full triangulated subcategory closed under direct summands. Now let $\mathcal{C}$ and $\mathcal{D}$ be subcategories of $\mathcal{T}$. We denote by $\operatorname{thick}_{\mathcal{T}}^{1}(\mathcal{C})$ the full subcategory of $\mathcal{T}$ consisting of all the direct summands of finite direct sums of shifts of objects in $\mathcal{C}$. Furthermore, we denote by $\mathcal{C} * \mathcal{D}$ the full subcategory of $\mathcal{T}$ consisting of objects $M$ such that there exists a distinguished triangle

$$
C \rightarrow M \rightarrow D \rightarrow \Sigma C
$$

in $\mathcal{T}$, with $C \in \mathcal{C}$ and $D \in \mathcal{D}$. Now for each $n \geqslant 2$, define inductively thick $\operatorname{T}_{\mathcal{T}}^{n}(\mathcal{C})$ to be thick $1_{\mathcal{T}}^{1}\left(\operatorname{thick}_{\mathcal{T}}^{n-1}(\mathcal{C}) * \operatorname{thick}_{\mathcal{T}}^{1}(\mathcal{C})\right)$, and denote $\bigcup_{n=1}^{\infty} \operatorname{thick}_{\mathcal{T}}^{n}(\mathcal{C})$ by thick $\mathcal{T}(\mathcal{C})$. This is the smallest thick subcategory of $\mathcal{T}$ containing $\mathcal{C}$.

The aim of this paper is to study the vanishing of cohomology in triangulated categories satisfying a certain finite generation hypothesis. This finite generation hypothesis is expressed in terms of the graded center $Z^{*}(\mathcal{T})$ of our triangulated category $\mathcal{T}$. Recall therefore that for an integer $n \in \mathbb{Z}$, the degree $n$ component $Z^{n}(\mathcal{T})$ is the set of natural transformations Id $\stackrel{f}{\rightarrow} \Sigma^{n}$ satisfying $f_{\Sigma X}=(-1)^{n} \Sigma f_{X}$ for each object $X \in \mathcal{T}$. This turns the graded center $Z^{*}(\mathcal{T})$ into a graded-commutative ring. For a homogeneous central element $f$ and objects $X, Y \in \mathcal{T}$, consider the graded group $\operatorname{Hom}_{\mathcal{T}}^{*}(X, Y)=\oplus_{i \in \mathbb{Z}} \operatorname{Hom}_{\mathcal{T}}\left(X, \Sigma^{i} Y\right)$. The element $f$ acts from the right on this graded group via the morphism $X \stackrel{f_{X}}{\longrightarrow} \Sigma^{n} X$, and from the left via the morphism $Y \stackrel{f_{Y}}{\longrightarrow} \Sigma^{n} Y$. Namely, given a morphism $g \in \operatorname{Hom}_{\mathcal{T}}\left(X, \Sigma^{m} Y\right)$, the scalar product $g f$ is the composition $X \stackrel{f_{X}}{\longrightarrow} \Sigma^{n} X \stackrel{\Sigma^{n} g}{\longrightarrow} \Sigma^{m+n} Y$, whereas $f g$ is the composition $X \stackrel{g}{\rightarrow} \Sigma^{m} Y \stackrel{\Sigma^{m} f_{Y}}{\longrightarrow} \Sigma^{m+n} Y$. However, since Id $\stackrel{f}{\rightarrow} \Sigma^{n}$ is a natural transformation, the diagram

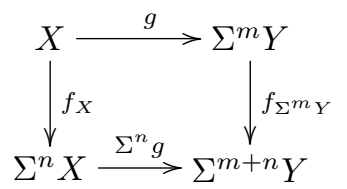

commutes, and so since $f_{\Sigma^{m} Y}$ equals $(-1)^{m n} \Sigma^{m} f_{Y}$ we see that $g f=(-1)^{m n} f g$. This shows that $Z^{*}(\mathcal{T})$ acts graded-commutatively on $\operatorname{Hom}_{\mathcal{T}}^{*}(X, Y)$ for all objects $X$ and $Y$ in $\mathcal{T}$. For further details on the graded center and its action on the cohomology groups, see $[\mathbf{1 4}]$. 
Now let $R=\oplus_{i=0}^{\infty} R_{i}$ be a graded-commutative ring, that is, for homogeneous elements $r_{1}, r_{2} \in R$ the equality $r_{1} r_{2}=(-1)^{\left|r_{1}\right|\left|r_{2}\right|} r_{2} r_{1}$ holds (where $|r|$ denotes the degree of a homogeneous element $r \in R$ ). We say that $R$ acts centrally on $\mathcal{T}$ if there exists a graded ring homomorphism $R \rightarrow Z^{*}(\mathcal{T})$. If this is the case, then for every object $X \in \mathcal{T}$ there is a graded ring homomorphism $R \stackrel{\varphi_{X}}{\longrightarrow} \operatorname{Hom}_{\mathcal{T}}^{*}(X, X)$ with the following property: for all objects $Y \in \mathcal{T}$ the scalar actions from $R$ on $\operatorname{Hom}_{\mathcal{T}}^{*}(X, Y)$ via $\varphi_{X}$ and $\varphi_{Y}$ are graded equivalent, i.e.,

$$
\varphi_{Y}(r) f=(-1)^{|r||f|} f \varphi_{X}(r)
$$

for all homogeneous elements $r \in R$ and $f \in \operatorname{Hom}_{\mathcal{T}}^{*}(X, Y)$. Following [6], we say that the $R$-module $\operatorname{Hom}_{\mathcal{T}}^{*}(X, Y)$ is eventually Noetherian, and write $\operatorname{Hom}_{\mathcal{T}}^{*}(X, Y) \in$ Noeth $R$, if there exists an integer $n_{0} \in \mathbb{Z}$ such that the $R$-module $\operatorname{Hom}_{\mathcal{T}}^{\geqslant n_{0}}(X, Y)$ is Noetherian. Moreover, we say that $\operatorname{Hom}_{\mathcal{T}}^{*}(X, Y)$ is eventually Noetherian of finite length, and write $\operatorname{Hom}_{\mathcal{T}}^{*}(X, Y) \in \operatorname{Noeth}^{\mathrm{fl}} R$, if $\operatorname{Hom}_{\mathcal{T}}^{*}(X, Y) \in \operatorname{Noeth} R$, and there exists an integer $n_{0} \in \mathbb{Z}$ such that $\ell_{R_{0}}\left(\operatorname{Hom}_{\mathcal{T}}\left(X, \Sigma^{n} Y\right)\right)<\infty$ for each $n \geqslant n_{0}$. Note that if $\operatorname{Hom}_{\mathcal{T}}^{*}(X, Y)$ is eventually Noetherian (respectively, eventually Noetherian of finite length), then so is $\operatorname{Hom}_{\mathcal{T}}^{*}\left(X^{\prime}, Y^{\prime}\right)$ for all objects $X^{\prime} \in \operatorname{thick} \mathcal{T}(X)$ and $Y^{\prime} \in \operatorname{thick}_{\mathcal{T}}(Y)$. In particular, if our category $\mathcal{T}$ is finitely generated in the sense that there exists an object $G$ such that $\mathcal{T}=\operatorname{thick}_{\mathcal{T}}(G)$, then $\operatorname{Hom}_{\mathcal{T}}^{*}(X, Y) \in$ Noeth $R$ (respectively, $\operatorname{Hom}_{\mathcal{T}}^{*}(X, Y) \in \operatorname{Noeth}^{\mathrm{fl}} R$ ) for all $X, Y \in \mathcal{T}$ if and only if $\operatorname{Hom}_{\mathcal{T}}^{*}(G, G) \in$ Noeth $R$ (respectively, $\operatorname{Hom}_{\mathcal{T}}^{*}(G, G) \in \operatorname{Noeth}^{\mathrm{fl}} R$ ).

Definition 2.1. Given objects $X$ and $Y$ of $\mathcal{T}$, we define the complexity of the ordered pair $(X, Y)$ as

$$
\operatorname{cx}_{\mathcal{T}}(X, Y) \stackrel{\text { def }}{=} \operatorname{dim}_{R^{\mathrm{ev}}} \operatorname{Hom}_{\mathcal{T}}^{*}(X, Y),
$$

where $R^{\text {ev }}$ denotes the commutative graded subalgebra $\oplus_{i=0}^{\infty} R_{2 i}$ of $R$, and dim denotes the Krull dimension. We define the complexity $\operatorname{cx}_{\mathcal{T}} X$ of the single object $X$ as $\operatorname{cx} \mathcal{T} X \stackrel{\text { def }}{=} \operatorname{cx} \mathcal{T}(X, X)$.

As is seen from the definition, the complexity of a pair of objects depends on the ring acting centrally. Therefore, strictly speaking, the correct notation should be something like $\operatorname{cx}_{\mathcal{T}}^{R}(X, Y)$. However, by [11, Lemma 4.3], the complexity is independent of the ring when the cohomology is finitely generated. Namely, if $R$ and $S$ are graded-commutative rings acting centrally on $\mathcal{T}$, and $\operatorname{Hom}_{\mathcal{T}}^{*}(X, Y)$ belongs to both $\operatorname{Noeth}^{\mathrm{fl}} R$ and $\operatorname{Noeth}^{\mathrm{fl}} S$, then $\operatorname{dim}_{R^{\mathrm{ev}}} \operatorname{Hom}_{\mathcal{T}}^{*}(X, Y)=\operatorname{dim}_{S^{\text {ev }}} \operatorname{Hom}_{\mathcal{T}}^{*}(X, Y)$. We therefore omit the ring in the notation of complexity; when studying vanishing of cohomology in $\mathcal{T}$, we will only be dealing with objects $X, Y \in \mathcal{T}$ with the property that the $R$-module $\operatorname{Hom}_{\mathcal{T}}^{*}(X, Y)$ is eventually Noetherian of finite length.

The fact that we will only be dealing with objects having finitely generated cohomology motivates the choice of terminology. Namely, it follows from [11, Proposition 2.6] that if $\operatorname{Hom}_{\mathcal{T}}^{*}(X, Y) \in \operatorname{Noeth}^{\mathrm{fl}} R$, then the Krull dimension of the $R^{\mathrm{ev}}$-module $\operatorname{Hom}_{\mathcal{T}}^{*}(X, Y)$ equals the infimum of all non-negative integers $t$ with the following property: there exists a real number $a$ such that

$$
\ell_{R_{0}}\left(\operatorname{Hom}_{\mathcal{T}}\left(X, \Sigma^{n} Y\right)\right) \leqslant a n^{t-1}
$$

for $n \gg 0$. A priori, the complexity of a pair is not finite. However, finiteness holds when $\operatorname{Hom}_{\mathcal{T}}^{*}(X, Y)$ is eventually Noetherian of finite length. To see 
this, let $n_{0}$ be an integer such that the $R$-module $\operatorname{Hom}_{\mathcal{T}}^{\geqslant n_{0}}(X, Y)$ is Noetherian and $\ell_{R_{0}}\left(\operatorname{Hom}_{\mathcal{T}}\left(X, \Sigma^{n} Y\right)\right)<\infty$ for each $n \geqslant n_{0}$, and denote the ideal $\operatorname{Ann}_{R} \operatorname{Hom}_{\mathcal{T}}^{\geqslant n_{0}}(X, Y)$ in $R$ by $I$. By [11, Remark 2.1], the quotient ring $R / I$ is Noetherian, and its degree zero part $(R / I)_{0}$ is Artinian. The claim now follows from the Hilbert-Serre Theorem (cf. [1, Theorem 11.1]).

It follows from the above alternative description of complexity that if $X$ and $Y$ are objects of $\mathcal{T}$ with $\operatorname{Hom}_{\mathcal{T}}^{*}(X, Y) \in \operatorname{Noeth}^{\mathrm{fl}} R$, then $\operatorname{cx}_{\mathcal{T}}(X, Y)=0$ if and only if $\operatorname{Hom}_{\mathcal{T}}^{*}(X, Y)$ is eventually zero, that is, if $\operatorname{Hom}_{\mathcal{T}}\left(X, \Sigma^{n} Y\right)=0$ for $n \gg 0$. Now digress for a moment, and let $\Lambda$ be a ring. Then $\Lambda$ is said to satisfy Auslander's condition if for every finitely generated module $M$, there exists an integer $d_{M}$, depending only on $M$, satisfying the following: if $N$ is a finitely generated $\Lambda$-module and $\operatorname{Ext}_{\Lambda}^{n}(M, N)=0$ for $n \gg 0$, then $\operatorname{Ext}_{\Lambda}^{n}(M, N)=0$ for $n \geqslant d_{M}$. Such algebras were studied in [15]. Motivated by this, we say that a full subcategory $\mathcal{C}$ of $\mathcal{T}$ satisfies the left Auslander condition if for every object $X \in \mathcal{C}$, there exists an integer $d_{X}$, depending only on $X$, such that the following holds: if $\operatorname{Hom}_{\mathcal{T}}^{*}(X, Y)$ is eventually zero for some object $Y \in \mathcal{T}$, then $\operatorname{Hom}_{\mathcal{T}}\left(X, \Sigma^{n} Y\right)=0$ for $n \geqslant d_{X}$. It is easy to see that this holds if and only if for all objects $X \in \mathcal{C}$ and $Y \in \mathcal{T}$, the implication

$$
\operatorname{Hom}_{\mathcal{T}}\left(X, \Sigma^{n} Y\right)=0 \text { for } n \gg 0 \Longrightarrow \operatorname{Hom}_{\mathcal{T}}\left(X, \Sigma^{n} Y\right)=0 \text { for all } n \in \mathbb{Z}
$$

holds. Dually, we say that $\mathcal{C}$ satisfies the right Auslander condition if the implication

$$
\operatorname{Hom}_{\mathcal{T}}\left(Y, \Sigma^{n} X\right)=0 \text { for } n \gg 0 \Longrightarrow \operatorname{Hom}_{\mathcal{T}}\left(Y, \Sigma^{n} X\right)=0 \text { for all } n \in \mathbb{Z}
$$

holds for all $X \in \mathcal{C}$ and $Y \in \mathcal{T}$. Note that if an object $X \in \mathcal{T}$ belongs to a subcategory of $\mathcal{T}$ satisfying either the left or the right Auslander condition, then $\operatorname{Hom}_{\mathcal{T}}^{*}(X, X)$ is eventually zero if and only if $X=0$. Note also that, even if a ring $\Lambda$ satisfies Auslander's condition, its bounded derived category does not (neither left nor right).

\section{Vanishing of cohomology}

In this section, we prove that if $\operatorname{Hom}_{\mathcal{T}}^{*}(X, X)$ is eventually Noetherian of finite length over some ring acting centrally on $\mathcal{T}$, then $\operatorname{Hom}_{\mathcal{T}}^{*}(X, Y)$ (and $\left.\operatorname{Hom}_{\mathcal{T}}^{*}(Y, X)\right)$ is zero if it contains a finite vanishing gap of a certain length. We start with a result which is the key ingredient in the main theorem. It shows that we can always reduce the complexity of a given object $X$, in the sense that there exists a triangle containing $X$, one of its shifts $\Sigma^{n} X$, and a third object whose complexity is less than that of $X$. Recall first the following notion, introduced in [9]. Let $R$ be a graded-commutative ring acting centrally on $\mathcal{T}$, and let $X \in \mathcal{T}$ be an object. Then, given a homogeneous element $r \in R$, we can complete the map $X \stackrel{\varphi_{X}(r)}{\longrightarrow} \Sigma^{|r|} X$ into a triangle

$$
X \stackrel{\varphi_{X}(r)}{\longrightarrow} \Sigma^{|r|} X \rightarrow X / / r \rightarrow \Sigma X .
$$

The object $X / / r$ is well defined up to isomorphism and is called a Koszul object of $r$ on $X$.

Proposition 3.1. Let $R$ be a graded-commutative ring acting centrally on $\mathcal{T}$, and let $X \in \mathcal{T}$ be an object such that $\operatorname{Hom}_{\mathcal{T}}^{*}(X, X) \in \operatorname{Noeth}^{\mathrm{fl}} R$. Then if $\operatorname{cx}_{\mathcal{T}} X$ is nonzero, 
there exists a homogeneous element $r \in R$, of positive degree, whose Koszul object $X / / r$ in the triangle

$$
X \stackrel{\varphi_{X}(r)}{\longrightarrow} \Sigma^{|r|} X \rightarrow X / / r \rightarrow \Sigma X
$$

satisfies $\mathrm{cx}_{\mathcal{T}} X / / r=\mathrm{cx}_{\mathcal{T}} X-1$.

Proof. Suppose $\mathrm{cx}_{\mathcal{T}} X>0$. By [11, Lemma 2.5], there exists an integer $i_{0}$ and a homogeneous element $r \in R$, of positive degree, such that scalar multiplication

$$
\operatorname{Hom}_{\mathcal{T}}\left(X, \Sigma^{i} X\right) \stackrel{\cdot r}{\rightarrow} \operatorname{Hom}_{\mathcal{T}}\left(X, \Sigma^{i+|r|} X\right)
$$

is injective for $i \geqslant i_{0}$. Applying $\operatorname{Hom}_{\mathcal{T}}(X,-)$ to the triangle

$$
X \stackrel{\varphi_{X}(r)}{\longrightarrow} \Sigma^{|r|} X \rightarrow X / / r \rightarrow \Sigma X,
$$

we obtain a long exact sequence

$$
\cdots \rightarrow \operatorname{Hom}_{\mathcal{T}}\left(X, \Sigma^{i} X\right) \stackrel{\cdot(-1)^{i} r}{\longrightarrow} \operatorname{Hom}_{\mathcal{T}}\left(X, \Sigma^{i+|r|} X\right) \rightarrow \operatorname{Hom}_{\mathcal{T}}\left(X, \Sigma^{i} X / / r\right) \rightarrow \cdots
$$

in cohomology. This long exact sequence induces a short exact sequence

$$
0 \rightarrow \operatorname{Hom}_{\mathcal{T}}^{\geqslant i_{0}}(X, X) \stackrel{\cdot r}{\rightarrow} \operatorname{Hom}_{\mathcal{T}}^{\geqslant i_{0}+|r|}(X, X) \rightarrow \operatorname{Hom}_{\mathcal{T}}^{\geqslant i_{0}}(X, X / / r) \rightarrow 0
$$

of eventually Noetherian $R$-modules of finite length. A standard argument (see, for example, [10, Proof of Theorem 3.2]) then gives $\operatorname{cx}_{\mathcal{T}}(X, X / / r)=\operatorname{cx}_{\mathcal{T}} X-1$. From this exact sequence we also see that the element $r$ annihilates $\operatorname{Hom}_{\mathcal{T}}^{\geqslant i_{0}}(X, X / / r)$. Therefore, when applying $\operatorname{Hom}_{\mathcal{T}}(-, X / / r)$ to our triangle, we obtain a short exact sequence

$$
0 \rightarrow \operatorname{Hom}_{\mathcal{T}}^{\geqslant i_{0}+|r|}(X, X / / r) \rightarrow \operatorname{Hom}_{\mathcal{T}}^{\geqslant i_{0}+|r|+1}(X / / r, X / / r) \rightarrow \operatorname{Hom}_{\mathcal{T}}^{\geqslant i_{0}+1}(X, X / / r) \rightarrow 0
$$

of eventually Noetherian $R$-modules of finite length. This gives the inequality $\operatorname{cx}_{\mathcal{T}} X / / r \leqslant \operatorname{cx}_{\mathcal{T}}(X, X / / r)$.

Since $\operatorname{Hom}_{\mathcal{T}}^{*}(X, X / / r)$ is eventually Noetherian, there exists an integer $n_{0}$ such that the $R$-module $\operatorname{Hom}_{\mathcal{T}}^{\geqslant n_{0}}(X, X / / r)$ is finitely generated. The $R$-scalar action factors through the ring homomorphism $\varphi_{X / / r}$, and therefore $\operatorname{Hom}_{\mathcal{T}} \gtrless_{0}(X, X / / r)$ is also finitely generated as a module over $\operatorname{Hom}_{\mathcal{T}}^{*}(X / / r, X / / r)$. Consequently, the rate of growth of the sequence $\left\{\ell_{R_{0}}\left(\operatorname{Hom}_{\mathcal{T}}\left(X, \Sigma^{n} X / / r\right)\right)\right\}_{n=1}^{\infty}$ is at most the rate of growth of $\left\{\ell_{R_{0}}\left(\operatorname{Hom}_{\mathcal{T}}\left(X / / r, \Sigma^{n} X / / r\right)\right)\right\}_{n=1}^{\infty}$, i.e., $\operatorname{cx}_{\mathcal{T}}(X, X / / r) \leqslant \operatorname{cx}_{\mathcal{T}} X / / r$.

Now let $X$ be an object of $\mathcal{T}$, and let $\left(r_{1}, \ldots, r_{c}\right)$ be a sequence of homogeneous elements belonging to a graded-commutative ring $R$ acting centrally on $\mathcal{T}$, with $c \geqslant 2$. Then we define the Koszul object $X / /\left(r_{1}, \ldots, r_{c}\right)$ inductively as

$$
X / /\left(r_{1}, \ldots, r_{c}\right) \stackrel{\text { def }}{=}\left(X / /\left(r_{1}, \ldots, r_{c-1}\right)\right) / / r_{c} .
$$

Suppose the complexity of $X$ is nonzero and finite, say $\mathrm{cx}_{\mathcal{T}} X=c$. We say that a sequence $\left(r_{1}, \ldots, r_{c}\right)$ of positive degree homogeneous elements of $R$ reduces the complexity of $X$ if $\operatorname{cx}_{\mathcal{T}} X / /\left(r_{1}, \ldots, r_{i}\right)=c-i$ for $1 \leqslant i \leqslant c$. Such a sequence gives rise 
to triangles

$$
\begin{gathered}
X \rightarrow \Sigma^{\left|r_{1}\right|} X \rightarrow X_{1} \rightarrow \Sigma X \\
X_{1} \rightarrow \Sigma^{\left|r_{2}\right|} X_{1} \rightarrow X_{2} \rightarrow \Sigma X_{1} \\
\vdots \\
X_{c-1} \rightarrow \Sigma^{\left|r_{c}\right|} X_{c-1} \rightarrow X_{c} \rightarrow \Sigma X_{c-1}
\end{gathered}
$$

in which $X_{i}=X / /\left(r_{1}, \ldots, r_{i}\right)$ for $1 \leqslant i \leqslant c$. Note that the sequence also reduces the complexity of $\Sigma^{n} X$ for any $n \in \mathbb{Z}$, since the complexity of an object $Y$ equals that of $\Sigma^{n} Y$ for all objects $Y$ in $\mathcal{T}$.

The main results of this section show that we can detect vanishing of cohomology from finite vanishing gaps. The length of these gaps depends on the sum of the degrees of the elements in a sequence reducing the complexity of an object. We therefore make the following definition.

Definition 3.2. Given an object $X$ of $\mathcal{T}$ of finite complexity $c$, we define $d_{\mathcal{T}}^{R}(X)$ as follows: if $c=0$, then $d_{\mathcal{T}}^{R}(X)=0$, and if $c>0$, then

$$
d_{\mathcal{T}}^{R}(X)=\inf \left\{\left|r_{1}\right|+\cdots+\left|r_{c}\right| \mid\left(r_{1}, \ldots, r_{c}\right) \text { reduces the complexity of } X\right\} .
$$

Note that the inequality $d_{\mathcal{T}}^{R}(X) \geqslant \operatorname{cx}_{\mathcal{T}} X$ always holds. Moreover, if there does not exist a sequence reducing the complexity of $X$, then $d_{\mathcal{T}}^{R}(X)$ is infinite.

If the complexity of an object in $\mathcal{T}$ is finite, then does there exist a sequence in $R$ reducing it? Suppose the $R$-module $\operatorname{Hom}_{\mathcal{T}}^{*}(X, X)$ is eventually Noetherian of finite length, and denote the complexity of $X$ by $c$. As we have seen, the finiteness condition implies that $c$ is finite. If $c>0$, then the previous result guarantees the existence of a homogeneous element $r_{1} \in R$, of positive degree, such that $\mathrm{cx}_{\mathcal{T}} X / / r_{1}=$ $c-1$. Since $X / / r_{1}$ belongs to thick $\mathcal{T}(X)$, the $R$-module $\operatorname{Hom}_{\mathcal{T}}^{*}\left(X / / r_{1}, X / / r_{1}\right)$ is also eventually Noetherian of finite length. Therefore, if $c-1>0$, then we may use the above result again; there exists a homogeneous element $r_{2} \in R$, of positive degree, such that $\mathrm{cx}_{\mathcal{T}} X / /\left(r_{1}, r_{2}\right)=c-2$. Continuing like this, we obtain a sequence $\left(r_{1}, \ldots, r_{c}\right)$ of homogeneous elements of $R$, all of positive degree, with the property that $\operatorname{cx}_{\mathcal{T}} X / /\left(r_{1}, \ldots, r_{i}\right)=c-i$ for $1 \leqslant i \leqslant c$. Thus when $\operatorname{Hom}_{\mathcal{T}}^{*}(X, X)$ is eventually Noetherian of finite length, then a reducing sequence exists if $\operatorname{cx}_{\mathcal{T}} X>0$, and consequently $d_{\mathcal{T}}^{R}(X)$ is finite. We record this in the following lemma.

Lemma 3.3. If $X$ is an object of $\mathcal{T}$ with $\operatorname{Hom}_{\mathcal{T}}^{*}(X, X) \in \operatorname{Noeth}^{\mathrm{fl}} R$ for some gradedcommutative ring $R$ acting centrally on $\mathcal{T}$, then $d_{\mathcal{T}}^{R}(X)$ is finite.

Note that a sequence reducing the complexity of an object is not unique in general. To see this, suppose that $\operatorname{Hom}_{\mathcal{T}}^{*}(X, X)$ belongs to Noeth ${ }^{\mathrm{fl}} R$ for some gradedcommutative ring $R$ acting centrally on $\mathcal{T}$ and that $\mathrm{cx}_{\mathcal{T}} X>0$. By [11, Lemma 2.5], there exists a homogeneous element $r$ of $R$, of positive degree, such that scalar multiplication

$$
\operatorname{Hom}_{\mathcal{T}}\left(X, \Sigma^{i} X\right) \stackrel{\cdot r}{\longrightarrow} \operatorname{Hom}_{\mathcal{T}}\left(X, \Sigma^{i+|r|} X\right)
$$

is injective for $i \gg 0$. The proof of Proposition 3.1 shows that $\mathrm{cx}_{\mathcal{T}} X / / r=\mathrm{cx}_{\mathcal{T}} X-1$, and so from the discussion prior to Lemma 3.3, we see that $r$ is the first element 
in a sequence reducing the complexity of $X$. However, for any number $n$, scalar multiplication

$$
\operatorname{Hom}_{\mathcal{T}}\left(X, \Sigma^{i} X\right) \stackrel{\cdot r^{n}}{\longrightarrow} \operatorname{Hom}_{\mathcal{T}}\left(X, \Sigma^{i+n|r|} X\right)
$$

is also injective for $i \gg 0$. Consequently, the element $r^{n}$ is also the first element in a sequence reducing the complexity of $X$.

We now prove our main result. It shows that, for two objects $X$ and $Y$ with $X$ satisfying the appropriate finiteness conditions, a large gap in $\operatorname{Hom}_{\mathcal{T}}^{*}(X, Y)$ cannot occur unless $\operatorname{Hom}_{\mathcal{T}}^{*}(X, Y)$ is actually zero.

Theorem 3.4. Let $X$ and $Y$ be objects of $\mathcal{T}$ with $\operatorname{Hom}_{\mathcal{T}}^{*}(X, X) \in \operatorname{Noeth}^{\mathrm{fl}} R$ for some graded-commutative ring $R$ acting centrally on $\mathcal{T}$, and suppose that the subcategory $\operatorname{thick}_{\mathcal{T}}(X)$ of $\mathcal{T}$ satisfies either the left or the right Auslander condition. Then the following are equivalent:

(i) There exists an integer $n \in \mathbb{Z}$ such that $\operatorname{Hom}_{\mathcal{T}}\left(X, \Sigma^{i} Y\right)=0$ for $n \leqslant i \leqslant n+$ $d_{\mathcal{T}}^{R}(X)-\operatorname{cx}_{\mathcal{T}} X$.

(ii) $\operatorname{Hom}_{\mathcal{T}}\left(X, \Sigma^{i} Y\right)=0$ for all $i \in \mathbb{Z}$.

Proof. We argue by induction on $c=\operatorname{cx}_{\mathcal{T}} X$ that (i) implies (ii). If $c$ is zero, then $\operatorname{Hom}_{\mathcal{T}}^{*}(X, X)$ is eventually zero, and so $X=0$ since thick $\mathcal{T}(X)$ satisfies either the left or the right Auslander condition. If $c>0$, then let $\left(r_{1}, \ldots, r_{c}\right)$ be a sequence reducing the complexity of $X$, with $\left|r_{1}\right|+\cdots+\left|r_{c}\right|=d_{\mathcal{T}}^{R}(X)$, and consider the triangle

$$
X \rightarrow \Sigma^{\left|r_{1}\right|} X \rightarrow X / / r_{1} \rightarrow \Sigma X .
$$

Applying $\operatorname{Hom}_{\mathcal{T}}(-, Y)$ to this triangle gives the long exact sequence

$$
\cdots \rightarrow \operatorname{Hom}_{\mathcal{T}}\left(X, \Sigma^{i-1} Y\right) \rightarrow \operatorname{Hom}_{\mathcal{T}}\left(X / / r_{1}, \Sigma^{i} Y\right) \rightarrow \operatorname{Hom}_{\mathcal{T}}\left(X, \Sigma^{i-\left|r_{1}\right|} Y\right) \rightarrow \cdots
$$

in cohomology, from which we see that $\operatorname{Hom}_{\mathcal{T}}\left(X / / r_{1}, \Sigma^{i} Y\right)=0$ for

$$
\left(n+\left|r_{1}\right|\right) \leqslant i \leqslant\left(n+\left|r_{1}\right|\right)+\left|r_{2}\right|+\cdots+\left|r_{c}\right|-(c-1) .
$$

The complexity of $X / / r_{1}$ is $c-1$, and the sequence $r_{2}, \ldots, r_{c}$ is a complexity reducing sequence for this object. Therefore, by the induction hypothesis and the fact that $\left|r_{2}\right|+\cdots+\left|r_{c}\right| \geqslant d_{\mathcal{T}}^{R}\left(X / / r_{1}\right)$, we conclude that $\operatorname{Hom}_{\mathcal{T}}\left(X / / r_{1}, \Sigma^{i} Y\right)=0$ for all $i \in \mathbb{Z}$. The long exact sequence then shows that $\operatorname{Hom}_{\mathcal{T}}\left(X, \Sigma^{i} Y\right)$ is isomorphic to $\operatorname{Hom}_{\mathcal{T}}\left(X, \Sigma^{i+\left|r_{1}\right|} Y\right)$ for all integers $i$, and from (i) we then see that $\operatorname{Hom}_{\mathcal{T}}\left(X, \Sigma^{i} Y\right)$ must vanish for all $i \in \mathbb{Z}$.

If we interchange the objects $X$ and $Y$ in the theorem, then the corresponding result of course holds. We state this without proof.

Theorem 3.5. Let $X$ and $Y$ be objects of $\mathcal{T}$ with $\operatorname{Hom}_{\mathcal{T}}^{*}(X, X) \in \operatorname{Noeth}^{\mathrm{fl}} R$ for some graded-commutative ring $R$ acting centrally on $\mathcal{T}$, and suppose that the subcategory thick $\mathcal{T}(X)$ of $\mathcal{T}$ satisfies either the left or the right Auslander condition. Then the following are equivalent:

(i) There exists an integer $n \in \mathbb{Z}$ such that $\operatorname{Hom}_{\mathcal{T}}\left(Y, \Sigma^{i} X\right)=0$ for $n \leqslant i \leqslant n+$ $d_{\mathcal{T}}^{R}(X)-\operatorname{cx}_{\mathcal{T}} X$.

(ii) $\operatorname{Hom}_{\mathcal{T}}\left(Y, \Sigma^{i} X\right)=0$ for all $i \in \mathbb{Z}$. 
We now use these results to study the vanishing of cohomology over Artin algebras. Let $\Lambda$ be a Noetherian ring, and denote the bounded derived category of finitely generated $\Lambda$-modules by $D^{b}(\Lambda)$. Furthermore, let $D^{\text {perf }}(\Lambda)$ be the thick subcategory of $D^{b}(\Lambda)$ generated by $\Lambda$; it consists of the perfect complexes, that is, objects isomorphic to bounded complexes of finitely generated projective $\Lambda$-modules. The stable derived category of $\Lambda$, denoted $D_{\mathrm{st}}^{b}(\Lambda)$, is the Verdier quotient

$$
D_{\text {st }}^{b}(\Lambda) \stackrel{\text { def }}{=} D^{b}(\Lambda) / D^{\text {perf }}(\Lambda)
$$

This is a triangulated category whose suspension functor corresponds to that in $D^{b}(\Lambda)$. Moreover, by [11, Remark 5.1], the central action of a graded-commutative ring $R$ on $D^{b}(\Lambda)$ carries over to $D_{\mathrm{st}}^{b}(\Lambda)$ via the ring homomorphism $Z^{*}\left(D^{b}(\Lambda)\right) \rightarrow$ $Z^{*}\left(D_{\mathrm{st}}^{b}(\Lambda)\right)$ induced by the natural quotient functor. Thus if $X$ and $Y$ are complexes in $D^{b}(\Lambda)$, then the natural map

$$
\operatorname{Hom}_{D^{b}(\Lambda)}^{*}(X, Y) \rightarrow \operatorname{Hom}_{D_{\mathrm{st}}^{b}(\Lambda)}^{*}(X, Y)
$$

is an $R$-module homomorphism. If $\Lambda$ is also Gorenstein, that is, if the injective dimension of $\Lambda$ both as a left and as a right module over itself is finite, then by [13, Corollary 6.3.4] this homomorphism is eventually bijective. That is, if $\Lambda$ is a Noetherian Gorenstein ring, then the natural map

$$
\operatorname{Hom}_{D^{b}(\Lambda)}\left(X, \Sigma^{n} Y\right) \rightarrow \operatorname{Hom}_{D_{\mathrm{st}}^{b}(\Lambda)}\left(X, \Sigma^{n} Y\right)
$$

is bijective for $n \gg 0$. Consequently, for any complexes $X$ and $Y$ in $D^{b}(\Lambda)$, if $\operatorname{Hom}_{D^{b}(\Lambda)}^{*}(X, Y)$ is an eventually Noetherian $R$-module of finite length, then so is $\operatorname{Hom}_{D_{\mathrm{st}}^{b}(\Lambda)}^{*}(X, Y)$.

Suppose $\Lambda$ is an Artin algebra, that is, the center $Z(\Lambda)$ of $\Lambda$ is a commutative Artin ring over which $\Lambda$ is finitely generated as a module. Denote by $\bmod \Lambda$ the category of finitely generated left $\Lambda$-modules. If $\Lambda$ is Gorenstein, then denote by $\operatorname{MCM}(\Lambda)$ the category of finitely generated maximal Cohen-Macaulay $\Lambda$-modules (cf. [3]), i.e.,

$$
\operatorname{MCM}(\Lambda)=\left\{M \in \bmod \Lambda \mid \operatorname{Ext}_{\Lambda}^{i}(M, \Lambda)=0 \text { for all } i>0\right\} .
$$

Its stable category $\operatorname{MCM}(\Lambda)$, which is obtained by factoring out all morphisms which factor through projective $\Lambda$-modules, is a triangulated category; for details, see [13] and [24]. Its shift functor is given by cokernels of left add $\Lambda$-approximations, the inverse shift is the usual syzygy functor. It follows from work by Buchweitz, Happel and Rickard (cf. [13], [25], $[\mathbf{2 9}]$ ) that $\underline{\operatorname{MCM}}(\Lambda)$ and the quotient category $D^{b}(\Lambda) / D^{\text {perf }}(\Lambda)$ are equivalent as triangulated categories. If $M$ and $N$ are maximal Cohen-Macaulay modules in $\bmod \Lambda$, then there is an isomorphism

$$
\operatorname{Ext}_{\Lambda}^{n}(M, N) \simeq \operatorname{Hom}_{\underline{\mathrm{MCM}(\Lambda)}}\left(\Omega_{\Lambda}^{n}(M), N\right)
$$

for every $n>0$. We use this isomorphism to prove the following result. It shows that when a certain finiteness condition holds, then the thick subcategory in $\underline{\operatorname{MCM}}(\Lambda)$ generated by a module satisfies both the left and the right Auslander condition.

Proposition 3.6. Let $\Lambda$ be an Artin Gorenstein algebra with Jacobson radical $\mathfrak{r}$, and let $M$ be a maximal Cohen-Macaulay module. If either $\operatorname{Ext}_{\Lambda}^{*}(M, \Lambda / \mathfrak{r})$ or $\operatorname{Ext}_{\Lambda}^{*}(\Lambda / \mathfrak{r}, M)$ belongs to $\mathrm{Noeth}^{\mathrm{fl}} R$ for some graded-commutative ring $R$ acting centrally on $D^{b}(\Lambda)$, 
then the subcategory thick $\mathrm{k}_{\mathrm{MCM}(\Lambda)}(M)$ of $\underline{\mathrm{MCM}}(\Lambda)$ satisfies both the left and the right Auslander condition.

Proof. Suppose that $\operatorname{Ext}_{\Lambda}^{*}(M, \Lambda / \mathfrak{r}) \in \operatorname{Noeth}^{\mathrm{fl}} R$. Let $X$ and $Y$ be maximal CohenMacaulay modules in $\bmod \Lambda$ with $X \in \operatorname{thick}_{\mathrm{MCM}(\Lambda)}(M)$, and suppose that $\operatorname{Hom}_{\underline{M C M}(\Lambda)}^{*}(X, Y)$ is eventually zero. We prove by induction on $\operatorname{cx}_{\underline{M C M}(\Lambda)} X$ that $\operatorname{Hom}_{\underline{\mathrm{MCM}}(\Lambda)}\left(X, \Sigma^{n} Y\right)=0$ for all $n \in \mathbb{Z}$.

Suppose $\operatorname{cx}_{\operatorname{MCM}(\Lambda)} X=0$. Induction on the length of any module $N \in \bmod \Lambda$ shows that since $\operatorname{Ext}_{\Lambda}^{*}(M, \Lambda / \mathfrak{r})$ belongs to $\operatorname{Noeth}^{\mathrm{fl}} R$, then so does $\operatorname{Ext}_{\Lambda}^{*}(M, N)$. Since $X \in \operatorname{thick}_{\mathrm{MCM}(\Lambda)}(M)$, the same holds for $\operatorname{Hom}_{\mathrm{MCM}(\Lambda)}^{*}(X, X)$. Therefore $\operatorname{Hom}_{\underline{\mathrm{MCM}}(\Lambda)}^{*}(X, X)$ is eventually zero, in particular $\operatorname{Ext}_{\Lambda}^{n}(X, X)=0$ for $n \gg 0$. Now consider the $R$-module $\operatorname{Ext}_{\Lambda}^{*}(X, \Lambda / \mathfrak{r})$. Since it belongs to $\operatorname{Noeth}^{\mathrm{fl}} R$, and the $R$ module structure factors through the ring homomorphism $R \stackrel{\varphi_{X}}{\longrightarrow} \operatorname{Ext}_{\Lambda}^{*}(X, X)$, we see that it must be eventually zero. The $\Lambda$-module $X$ therefore has finite projective dimension and is isomorphic to the zero object in $\operatorname{MCM}(\Lambda)$. Consequently, $\operatorname{Hom}_{\underline{M}(\Lambda)}\left(X, \Sigma^{n} Y\right)=0$ for all $n \in \mathbb{Z}$.

If $\operatorname{cx}_{\underline{M C M}(\Lambda)} X>0$, then let $r \in R$ be a homogeneous element of positive degree such that $\operatorname{cx}_{\underline{M C M}(\Lambda)} X / / r=\operatorname{cx}_{\underline{M C M}(\Lambda)} X-1$. Since $\operatorname{Hom}_{\underline{\underline{M C M}(\Lambda)}}^{*}(X, Y)$ is eventually zero, we see from the triangle

$$
X \rightarrow \Sigma^{|r|} X \rightarrow X / / r \rightarrow \Sigma X
$$

that the same holds for $\operatorname{Hom}_{\mathrm{MCM}(\Lambda)}^{*}(X / / r, Y)$. The Koszul object $X / / r$ belongs to thick $_{\underline{\mathrm{MCM}}(\Lambda)}(M)$, hence by induction $\operatorname{Hom}_{\underline{\mathrm{MCM}}(\Lambda)}\left(X / / r, \Sigma^{n} Y\right)=0$ for all $n \in \mathbb{Z}$. From the triangle we obtain the isomorphism

$$
\operatorname{Hom}_{\underline{\mathrm{MCM}}(\Lambda)}\left(X, \Sigma^{n} Y\right) \simeq \operatorname{Hom}_{\underline{\mathrm{MCM}}(\Lambda)}\left(X, \Sigma^{n+|r|} Y\right)
$$

for all integers $n$, and this implies that $\operatorname{Hom}_{\operatorname{MCM}(\Lambda)}\left(X, \Sigma^{n} Y\right)=0$ for all $n \in \mathbb{Z}$.

We have now proved that if $\operatorname{Ext}_{\Lambda}^{*}(M, \Lambda / \mathfrak{r})$ is an eventually Noetherian $R$-module of finite length, then the subcategory thick $\underline{\mathrm{MCM}}(\Lambda)_{(M)}(M) \underline{\mathrm{MCM}}(\Lambda)$ satisfies the left Auslander condition. Virtually the same proof shows that thick $\operatorname{MCM}_{\mathrm{M}(\Lambda)}(M)$ also satisfies the right Auslander condition. Moreover, an analogous proof shows that the same holds if the $R$-module $\operatorname{Ext}_{\Lambda}^{*}(\Lambda / \mathfrak{r}, M)$ is eventually Noetherian of finite length.

Before proving the next result, we recall the following: Let $\Lambda$ be an Artin algebra with Jacobson radical $\mathfrak{r}$, and let $M \in \bmod \Lambda$ be a module with minimal projective and injective resolutions

$$
\cdots \rightarrow P_{2} \rightarrow P_{1} \rightarrow P_{0} \rightarrow M \rightarrow 0
$$

and

$$
0 \rightarrow M \rightarrow I^{0} \rightarrow I^{1} \rightarrow I^{2} \rightarrow \cdots,
$$

respectively. Then the complexity and plexity of $M$, denoted $\operatorname{cx}_{\Lambda} M$ and $\operatorname{px}_{\Lambda} M$, respectively, are defined as

$$
\begin{aligned}
& \operatorname{cx}_{\Lambda} M \stackrel{\text { def }}{=} \inf \left\{t \in \mathbb{N} \cup\{0\} \mid \exists a \in \mathbb{R} \text { such that } \ell_{Z(\Lambda)}\left(P_{n}\right) \leqslant a n^{t-1} \text { for } n \gg 0\right\}, \\
& \operatorname{px}_{\Lambda} M \stackrel{\text { def }}{=} \inf \left\{t \in \mathbb{N} \cup\{0\} \mid \exists a \in \mathbb{R} \text { such that } \ell_{Z(\Lambda)}\left(I^{n}\right) \leqslant a n^{t-1} \text { for } n \gg 0\right\},
\end{aligned}
$$

where $Z(\Lambda)$ is the center of $\Lambda$. Now let $R$ be a graded-commutative ring acting centrally on $D^{b}(\Lambda)$. As we saw in the beginning of the previous proof, if the $R$-module 
$\operatorname{Ext}_{\Lambda}^{*}(M, \Lambda / \mathfrak{r})$ is eventually Noetherian of finite length, then so is $\operatorname{Ext}_{\Lambda}^{*}(M, N)$ for any $\Lambda$-module $N$. In particular, this holds for $\operatorname{Ext}_{\Lambda}^{*}(M, M)$, and $\mathrm{cx}_{\Lambda} M$ coincides with $\mathrm{cx}_{D^{b}(\Lambda)} M$ (cf. [11, Lemma 4.1]). Therefore there exists a sequence $\left(r_{1}, \ldots, r_{\mathrm{cx}_{\Lambda} M}\right)$ of homogeneous elements of $R$, all of positive degree, reducing the complexity of $M$ as an object in $D^{b}(\Lambda)$. It follows immediately that $d_{D^{b}(\Lambda)}^{R}(M)$ is finite and that $d_{D^{b}(\Lambda)}^{R}(M) \geqslant \operatorname{cx}_{\Lambda} M$.

Similarly, if the $R$-module $\operatorname{Ext}_{\Lambda}^{*}(\Lambda / \mathfrak{r}, M)$ is eventually Noetherian of finite length, then $\operatorname{px}_{\Lambda} M=\operatorname{cx}_{D^{b}(\Lambda)} M$. In this case, there exists a homogeneous sequence in $R$ of length $\mathrm{px}_{\Lambda} M$ reducing the complexity of $M$ as an object in $D^{b}(\Lambda)$. As above, it follows that $d_{D^{b}(\Lambda)}^{R}(M)$ is finite and that $d_{D^{b}(\Lambda)}^{R}(M) \geqslant \mathrm{px}_{\Lambda} M$. Using this and Proposition 3.6, we obtain the following vanishing results on cohomology over Gorenstein algebras. We prove only the first of these results; the proof of the other result is similar.

Theorem 3.7. Let $\Lambda$ be an Artin Gorenstein algebra with Jacobson radical $\mathfrak{r}$, and let $M \in \bmod \Lambda$ be a maximal Cohen-Macaulay module. Suppose $\operatorname{Ext}_{\Lambda}^{*}(M, \Lambda / \mathfrak{r}) \in$ Noeth $^{\mathrm{fl}} R$ for some graded-commutative ring $R$ acting centrally on $D^{b}(\Lambda)$.

(i) For any $N \in \bmod \Lambda$, the following are equivalent:

(a) There exists a number $n>$ id $\Lambda$ such that $\operatorname{Ext}_{\Lambda}^{i}(M, N)=0$ for $n \leqslant i \leqslant n+$ $d_{D^{b}(\Lambda)}^{R}(M)-\operatorname{cx}_{\Lambda} M$.

(b) $\operatorname{Ext}_{\Lambda}^{i}(M, N)=0$ for all $i>$ id $\Lambda$.

(ii) For any $N \in \bmod \Lambda$, the following are equivalent:

(a) There exists a number $n>$ id $\Lambda$ such that $\operatorname{Ext}_{\Lambda}^{i}(N, M)=0$ for $n \leqslant i \leqslant n+$ $d_{D^{b}(\Lambda)}^{R}(M)-\mathrm{cx}_{\Lambda} M$.

(b) $\operatorname{Ext}_{\Lambda}^{i}(N, M)=0$ for all $i>\operatorname{id} \Lambda$.

Proof. By $[\mathbf{2}$, Theorem 1.8], there exists an exact sequence

$$
0 \rightarrow Q \rightarrow C \rightarrow N \rightarrow 0
$$

in $\bmod \Lambda$, in which $Q$ has finite projective dimension and $C$ is maximal CohenMacaulay. Since $Q$ also has finite injective dimension and id $Q$ is at most id $\Lambda$, there are isomorphisms $\operatorname{Ext}_{\Lambda}^{i}(M, N) \simeq \operatorname{Ext}_{\Lambda}^{i}(M, C)$ for $i>$ id $\Lambda$. Moreover, the module $\Omega_{\Lambda}^{\text {id } \Lambda}(N)$ is maximal Cohen-Macaulay, and $\operatorname{Ext}_{\Lambda}^{i}(N, M) \simeq \operatorname{Ext}_{\Lambda}^{i-\operatorname{id} \Lambda}\left(\Omega_{\Lambda}^{\text {id } \Lambda}(N), M\right)$ for $i>$ id $\Lambda$. We may therefore, without loss of generality, assume that $N$ itself is maximal Cohen-Macaulay, and replace id $\Lambda$ by 0 in the statements. The implications now follow from Theorem 3.4, Theorem 3.5, Proposition 3.6 and the fact that $\operatorname{Ext}_{\Lambda}^{i}(X, Y) \simeq \operatorname{Hom}_{\mathrm{MCM}(\Lambda)}\left(X, \Sigma^{i} Y\right)$ when $X$ and $Y$ are maximal Cohen-Macaulay and $i>0$.

Theorem 3.8. Let $\Lambda$ be an Artin Gorenstein algebra with Jacobson radical $\mathfrak{r}$, and let $M \in \bmod \Lambda$ be a maximal Cohen-Macaulay module. Suppose $\operatorname{Ext}_{\Lambda}^{*}(\Lambda / \mathfrak{r}, M) \in$ Noeth $^{\mathrm{fl}} R$ for some graded-commutative ring $R$ acting centrally on $D^{b}(\Lambda)$.

(i) For any $N \in \bmod \Lambda$, the following are equivalent:

(a) There exists a number $n>$ id $\Lambda$ such that $\operatorname{Ext}_{\Lambda}^{i}(M, N)=0$ for $n \leqslant i \leqslant n+$ $d_{D^{b}(\Lambda)}^{R}(M)-\mathrm{px}_{\Lambda} M$.

(b) $\operatorname{Ext}_{\Lambda}^{i}(M, N)=0$ for all $i>\operatorname{id} \Lambda$. 
(ii) For any $N \in \bmod \Lambda$, the following are equivalent:

(a) There exists a number $n>$ id $\Lambda$ such that $\operatorname{Ext}_{\Lambda}^{i}(N, M)=0$ for $n \leqslant i \leqslant n+$ $d_{D^{b}(\Lambda)}^{R}(M)-\mathrm{px}_{\Lambda} M$.

(b) $\operatorname{Ext}_{\Lambda}^{i}(N, M)=0$ for all $i>$ id $\Lambda$.

We end this section with an example.

Example 3.9. Let $k$ be a field and $G$ a finite group whose order is divisible by the characteristic of $k$. The group cohomology ring $\mathrm{H}^{*}(G, k)$ is graded-commutative and acts centrally on $D^{b}(k G)$. Moreover, by a classical result of Evens and Venkov (cf. [21], $[\mathbf{3 2}],[\mathbf{3 3}])$, the cohomology ring is Noetherian, and $\operatorname{Ext}_{k G}^{*}(M, N)$ is a finitely generated $\mathrm{H}^{*}(G, k)$-module for all $M$ and $N$ in $\bmod k G$. Therefore Theorem 3.7 and Theorem 3.8 apply to group algebras.

\section{Symmetry}

As seen in the above example, group algebras of finite groups are examples of Artin algebras having finitely generated cohomology. Commutative local complete intersection rings also have finitely generated cohomology. Namely, let $(A, \mathfrak{m}, k)$ be such a local ring of codimension $c$, i.e., $c=\operatorname{dim}_{k}\left(\mathfrak{m} / \mathfrak{m}^{2}\right)-\operatorname{dim} A$, and let $\widehat{A}$ denote its completion with respect to its maximal ideal $\mathfrak{m}$. It was shown in [4] that there exists a certain polynomial ring $\widehat{A}\left[\chi_{1}, \ldots, \chi_{c}\right]$ acting centrally on $D^{b}(\widehat{A})$, where the $\chi_{i}$ are the so-called Eisenbud operators. For all finitely generated $A$-modules $M$ and $N$, the $\widehat{A}\left[\chi_{1}, \ldots, \chi_{c}\right]$-module $\operatorname{Ext}_{\widehat{A}}^{*}(\widehat{M}, \widehat{N})$ is finitely generated. Consequently, vanishing results similar to those in the previous section also hold in this case.

A fascinating aspect of the vanishing of cohomology over both group algebras and commutative local complete intersections is symmetry. In [5] it was shown that for finitely generated modules $M$ and $N$ over a commutative local complete intersection $A$, the vanishing of $\operatorname{Ext}_{A}^{i}(M, N)$ for $i \gg 0$ implies the vanishing of $\operatorname{Ext}_{A}^{i}(N, M)$ for $i \gg 0$. The proof involves the theory of certain support varieties attached to each pair of $A$-modules. Denote by $c$ the codimension of $A$ and by $K$ the algebraic closure of its residue field. A cone $\mathrm{V}_{A}^{*}(M, N)$ in $K^{c}$ is associated to the ordered pair $(M, N)$, with the following properties:

$$
\begin{gathered}
\mathrm{V}_{A}^{*}(M, N)=\{0\} \Leftrightarrow \operatorname{Ext}_{A}^{i}(M, N)=0 \text { for } i \gg 0 \\
\mathrm{~V}_{A}^{*}(M, N)=\mathrm{V}_{A}^{*}(M, M) \cap \mathrm{V}_{A}^{*}(N, N) .
\end{gathered}
$$

The symmetry in the vanishing of cohomology follows immediately from these properties. As shown in [28, Corollary 4.8], group algebras of finite groups provide another class of examples where Ext-symmetry holds.

We shall see in this section that in general there is no symmetry in the vanishing of cohomology over an Artin algebra, even when the algebra is self-injective and has finitely generated cohomology in the sense of group algebras. But first, we study situations where symmetry holds. Let $k$ be a commutative Artin ring, and suppose that $\mathcal{T}$ is a Hom-finite triangulated $k$-category. In other words, for all objects $X, Y, Z \in \mathcal{T}$, 
the group $\operatorname{Hom}_{\mathcal{T}}(X, Y)$ is a $k$-module of finite length, and the composition

$$
\operatorname{Hom}_{\mathcal{T}}(Y, Z) \times \operatorname{Hom}_{\mathcal{T}}(X, Y) \rightarrow \operatorname{Hom}_{\mathcal{T}}(X, Z)
$$

is $k$-bilinear. Let $E$ be an injective envelope of $k$ as a module over itself, and denote the functor $\operatorname{Hom}_{k}(-, E)$ by $D$. A Serre functor on $\mathcal{T}$ is an equivalence $\mathcal{T} \stackrel{S}{\rightarrow} \mathcal{T}$ of $k$-categories, together with functorial isomorphisms

$$
\operatorname{Hom}_{\mathcal{T}}(X, Y) \simeq D \operatorname{Hom}_{\mathcal{T}}(Y, S X)
$$

of $k$-modules for all objects $X, Y \in \mathcal{T}$. Note that we do not require such a functor to be triangulated. By [12], such a functor is unique if it exists. Following [27], for an integer $d \in \mathbb{Z}$, the category $\mathcal{T}$ is said to be weakly $d$-Calabi-Yau if it admits a Serre functor which is isomorphic as a $k$-linear functor to $\Sigma^{d}$. If, in addition, this isomorphism is an isomorphism of triangle functors, then $\mathcal{T}$ is $d$-Calabi-Yau. However, we will only be dealing with weakly $d$-Calabi-Yau categories. When $\mathcal{T}$ is such a category, then for all objects $X, Y \in \mathcal{T}$ there is an isomorphism

$$
\operatorname{Hom}_{\mathcal{T}}(X, Y) \simeq D \operatorname{Hom}_{\mathcal{T}}\left(Y, \Sigma^{d} Y\right)
$$

of $k$-modules. It follows immediately that if this holds, then $\operatorname{Hom}_{\mathcal{T}}\left(X, \Sigma^{n} Y\right)=0$ for $n \gg 0$ if and only if $\operatorname{Hom}_{\mathcal{T}}\left(Y, \Sigma^{n} X\right)=0$ for $n \ll 0$.

Now let $\Lambda$ be an Artin Gorenstein algebra. Following [28], we say that $\Lambda$ is stably symmetric if $\underline{\operatorname{MCM}}(\Lambda)$ is weakly $d$-Calabi-Yau for some integer $d \in \mathbb{Z}$. It was shown in that paper that if $\Lambda$ in addition satisfies Auslander's condition, then symmetry holds in the vanishing of cohomology of $\Lambda$-modules. The following result shows that symmetry holds for modules with finitely generated cohomology.

Theorem 4.1. Let $\Lambda$ be a stably symmetric Artin Gorenstein algebra with Jacobson radical $\mathfrak{r}$. Let $M \in \bmod \Lambda$ be a module such that either $\operatorname{Ext}_{\Lambda}^{*}(M, \Lambda / \mathfrak{r})$ or $\operatorname{Ext}_{\Lambda}^{*}(\Lambda / \mathfrak{r}, M)$ belongs to Noeth $^{\mathrm{f}} R$ for some graded-commutative ring $R$ acting centrally on $D^{b}(\Lambda)$. Then for every $N \in \bmod \Lambda$, the following are equivalent:

(i) $\operatorname{Ext}_{\Lambda}^{i}(M, N)=0$ for $i \gg 0$.

(ii) $\operatorname{Ext}_{\Lambda}^{i}(M, N)=0$ for $i>$ id $\Lambda$.

(iii) $\operatorname{Ext}_{\Lambda}^{i}(N, M)=0$ for $i \gg 0$.

(iv) $\operatorname{Ext}_{\Lambda}^{i}(N, M)=0$ for $i>\operatorname{id} \Lambda$.

Proof. As in the proof of Theorem 3.7, there exists an exact sequence

$$
0 \rightarrow Q_{N} \rightarrow C_{N} \rightarrow N \rightarrow 0
$$

in $\bmod \Lambda$, in which $Q_{N}$ has finite projective (and injective) dimension, and $C_{N}$ is maximal Cohen-Macaulay. Thus there is an isomorphism $\operatorname{Ext}_{\Lambda}^{i}(M, N) \simeq$ $\operatorname{Ext}_{\Lambda}^{i-\operatorname{id} \Lambda}\left(\Omega_{\Lambda}^{\mathrm{id} \Lambda}(M), C_{N}\right)$ for every $i>\operatorname{id} \Lambda$. Moreover, since either $\operatorname{Ext}_{\Lambda}^{*}(M, \Lambda / \mathfrak{r})$ or $\operatorname{Ext}_{\Lambda}^{*}(\Lambda / \mathfrak{r}, M)$ belongs to $\operatorname{Noeth}^{\mathrm{ff}} R$, so do either $\operatorname{Ext}_{\Lambda}^{*}\left(\Omega_{\Lambda}^{\mathrm{id} \Lambda}(M), \Lambda / \mathfrak{r}\right)$ or $\operatorname{Ext}_{\Lambda}^{*}\left(\Lambda / \mathfrak{r}, \Omega_{\Lambda}^{\text {id } \Lambda}(M)\right)$. Therefore, as shown in the proof of Theorem 3.7, and by Theorem 3.8, the implication

$$
\operatorname{Ext}_{\Lambda}^{i}\left(\Omega_{\Lambda}^{\mathrm{id} \Lambda}(M), C_{N}\right)=0 \text { for } i \gg 0 \Rightarrow \operatorname{Ext}_{\Lambda}^{i}\left(\Omega_{\Lambda}^{\mathrm{id} \Lambda}(M), C_{N}\right)=0 \text { for } i>0
$$

holds, showing that (i) implies (ii). The proof that (iii) implies (iv) is similar: start 
with an exact sequence

$$
0 \rightarrow Q_{M} \rightarrow C_{M} \rightarrow M \rightarrow 0
$$

where $Q_{M}$ has finite projective/injective dimension, and $C_{M}$ is maximal CohenMacaulay.

By Theorem 3.6, the subcategory thick $\underline{\underline{M C M}(\Lambda)}\left(C_{M}\right)$ of $\underline{\operatorname{MCM}}(\Lambda)$ satisfies both the left and the right Auslander condition. Moreover, by assumption $\underline{\operatorname{MCM}}(\Lambda)$ is weakly $d$-Calabi-Yau for some integer $d \in \mathbb{Z}$. Therefore the equivalences

$$
\begin{aligned}
\operatorname{Ext}_{\Lambda}^{i}(M, N)=0 \text { for } i \gg 0 & \Leftrightarrow \operatorname{Ext}_{\Lambda}^{i}\left(C_{M}, C_{N}\right)=0 \text { for } i \gg 0 \\
& \Leftrightarrow \operatorname{Hom}_{\underline{\mathrm{MCM}}(\Lambda)}\left(C_{M}, \Sigma^{i} C_{N}\right)=0 \text { for } i \gg 0 \\
& \Leftrightarrow \operatorname{Hom}_{\underline{\mathrm{MCM}}(\Lambda)}\left(C_{M}, \Sigma^{i} C_{N}\right)=0 \text { for } i \in \mathbb{Z} \\
& \Leftrightarrow \operatorname{Hom}_{\underline{\mathrm{MCM}}(\Lambda)}\left(C_{N}, \Sigma^{i} C_{M}\right)=0 \text { for } i \in \mathbb{Z} \\
& \Leftrightarrow \operatorname{Hom}_{\underline{\mathrm{MCM}}(\Lambda)}\left(C_{N}, \Sigma^{i} C_{M}\right)=0 \text { for } i \gg 0 \\
& \Leftrightarrow \operatorname{Ext}_{\Lambda}^{i}\left(C_{N}, C_{M}\right)=0 \text { for } i \gg 0 \\
& \Leftrightarrow \operatorname{Ext}_{\Lambda}^{i}(N, M)=0 \text { for } i \gg 0
\end{aligned}
$$

hold, and the proof is complete.

For an Artin algebra $\Lambda$ with radical $\mathfrak{r}$, if $\operatorname{Ext}_{\Lambda}^{*}(\Lambda / \mathfrak{r}, \Lambda / \mathfrak{r}) \in \operatorname{Noeth}^{\mathrm{fl}} R$ for some graded-commutative ring $R$ acting centrally on $D^{b}(\Lambda)$, then $\operatorname{Ext}_{\Lambda}^{*}(M, N) \in \operatorname{Noeth}^{\mathrm{fl}} R$ for all modules $M, N \in \bmod \Lambda$. Moreover, if this holds, then $\Lambda$ is automatically Gorenstein by $[\mathbf{1 1}$, Proposition 5.6]. Consequently, we obtain the following "global version" of Theorem 4.1.

Theorem 4.2. Let $\Lambda$ be a stably symmetric Artin algebra with Jacobson radical $\mathfrak{r}$, and suppose that $\operatorname{Ext}_{\Lambda}^{*}(\Lambda / \mathfrak{r}, \Lambda / \mathfrak{r})$ belongs to Noeth ${ }^{\mathrm{fl}} R$ for some graded-commutative ring $R$ acting centrally on $D^{b}(\Lambda)$. Then for all modules $M, N \in \bmod \Lambda$, the following are equivalent:

(i) $\operatorname{Ext}_{\Lambda}^{i}(M, N)=0$ for $i \gg 0$.

(ii) $\operatorname{Ext}_{\Lambda}^{i}(M, N)=0$ for $i>\operatorname{id} \Lambda$.

(iii) $\operatorname{Ext}_{\Lambda}^{i}(N, M)=0$ for $i \gg 0$.

(iv) $\operatorname{Ext}_{\Lambda}^{i}(N, M)=0$ for $i>\operatorname{id} \Lambda$.

Next, we include a special case of this theorem. Recall that for a commutative Artin ring $k$, an Artin $k$-algebra $\Lambda$ is symmetric if there is an isomorphism $\Lambda \simeq \operatorname{Hom}_{k}(\Lambda, k)$ of $\Lambda$ - $\Lambda$-bimodules. Such an algebra is necessarily self-injective.

Corollary 4.3. Let $\Lambda$ be a symmetric Artin algebra with Jacobson radical $\mathfrak{r}$, and suppose that $\operatorname{Ext}_{\Lambda}^{*}(\Lambda / \mathfrak{r}, \Lambda / \mathfrak{r})$ belongs to $\operatorname{Noeth}^{\mathrm{fl}} R$ for some graded-commutative ring $R$ acting centrally on $D^{b}(\Lambda)$. Then for all modules $M, N \in \bmod \Lambda$, the following are equivalent:

(i) $\operatorname{Ext}_{\Lambda}^{i}(M, N)=0$ for $i \gg 0$.

(ii) $\operatorname{Ext}_{\Lambda}^{i}(M, N)=0$ for $i>0$.

(iii) $\operatorname{Ext}_{\Lambda}^{i}(N, M)=0$ for $i \gg 0$.

(iv) $\operatorname{Ext}_{\Lambda}^{i}(N, M)=0$ for $i>0$. 
Proof. By [28, Corollary 4.4], a symmetric Artin algebra is stably symmetric.

We turn now to a particular class of algebras having finitely generated cohomology in the sense of Theorem 4.2 and Corollary 4.3. Details concerning the following can be found in $[\mathbf{3 0}]$ and $[\mathbf{3 1}]$. Let $k$ be a field and $\Lambda$ a finite dimensional $k$-algebra, and denote the enveloping algebra $\Lambda \otimes_{k} \Lambda^{\mathrm{op}}$ of $\Lambda$ by $\Lambda^{\mathrm{e}}$. For $n \geqslant 0$, the $n$th Hochschild cohomology group of $\Lambda$, denoted $\operatorname{HH}^{n}(\Lambda)$, is the vector space $\operatorname{Ext}_{\Lambda^{\mathrm{e}}}^{n}(\Lambda, \Lambda)$. The graded vector space $\operatorname{HH}^{*}(\Lambda)=\operatorname{Ext}_{\Lambda^{\mathrm{e}}}^{*}(\Lambda, \Lambda)$ is a graded-commutative ring with Yoneda product, and for every $M \in \bmod \Lambda$ the tensor product $-\otimes_{\Lambda} M$ induces a homomorphism

$$
\mathrm{HH}^{*}(\Lambda) \stackrel{\varphi_{M}}{\longrightarrow} \operatorname{Ext}_{\Lambda}^{*}(M, M)
$$

of graded $k$-algebras. If $N \in \bmod \Lambda$ is another module and $\eta \in \operatorname{HH}^{*}(\Lambda)$ and $\theta \in$ $\operatorname{Ext}_{\Lambda}^{*}(M, N)$ are homogeneous elements, then the relation $\varphi_{N}(\eta) \circ \theta=(-1)^{|\eta||\theta|} \theta \circ$ $\varphi_{M}(\eta)$ holds, where "o" denotes the Yoneda product. Therefore the Hochschild cohomology ring $\mathrm{HH}^{*}(\Lambda)$ acts centrally on $D^{b}(\Lambda)$. In [16], the authors studied the case when $\operatorname{HH}^{*}(\Lambda)$ is Noetherian and $\operatorname{Ext}_{\Lambda}^{*}(\Lambda / \mathfrak{r}, \Lambda / \mathfrak{r})$ is a finitely generated $\operatorname{HH}^{*}(\Lambda)$ module. This is a special case of the finiteness conditions assumed in Theorem Theorem 4.2 and Corollary 4.3.

Suppose now that $\Lambda / \mathfrak{r} \otimes_{k} \Lambda / \mathfrak{r}$ is semisimple, as happens, for example, when $k$ is algebraically closed. Furthermore, suppose that $\Lambda$ is a periodic algebra, that is, there exists a number $p>0$ such that $\Lambda$ is isomorphic to $\Omega_{\Lambda^{\mathrm{e}}}^{p}(\Lambda)$ as a left $\Lambda^{\mathrm{e}}$-module (i.e., as a bimodule). By [17], [18] and [20], this happens, for example, when $\Lambda$ is a self-injective Nakayama algebra, a Möbius algebra or a preprojective algebra (see also [19]). Moreover, by [22, Theorem 1.4], periodic algebras over algebraically closed fields are necessarily self-injective. Letting $Q_{n}$ denote the $n$th module in the minimal projective $\Lambda^{\mathrm{e}}$-resolution of $\Lambda$, we have an exact sequence

$$
0 \rightarrow \Lambda \rightarrow Q_{p-1} \rightarrow \cdots \rightarrow Q_{0} \rightarrow \Lambda \rightarrow 0
$$

of bimodules, and we denote this by $\mu$. This extension is an element of $\operatorname{HH}^{p}(\Lambda)$. If $\theta$ is an element of $\operatorname{HH}^{n}(\Lambda)$ for some $n>p$, then $\theta=\bar{\theta} \mu^{i}$ for some $i$ and a homogeneous element $\bar{\theta}$ of degree not more than $p$. Hence the Hochschild cohomology ring $\operatorname{HH}^{*}(\Lambda)$ is generated over $\mathrm{HH}^{0}(\Lambda)$ by the finite set of $k$-generators in $\mathrm{HH}^{1}(\Lambda), \ldots, \mathrm{HH}^{p}(\Lambda)$ and therefore is Noetherian. If $S$ is a simple non-projective $\Lambda$-module, then $\mu \otimes_{\Lambda} S$ is the beginning of the minimal projective resolution of $S$, since $\Lambda / \mathfrak{r} \otimes_{k} \Lambda / \mathfrak{r}$ is semisimple. Therefore $S$ must be periodic with period dividing $p$. If $N$ is any finitely generated $\Lambda$-module and $\omega$ is an element of $\operatorname{Ext}_{\Lambda}^{n}(S, N)$ for some $n>p$, then, as above, $\omega=$ $\bar{\omega}\left(\mu \otimes_{\Lambda} S\right)$ for some element $\bar{\omega} \in \operatorname{Ext}_{\Lambda}^{m}(S, N)$ with $m \leqslant p$. Therefore $\operatorname{Ext}_{\Lambda}^{*}(S, N)$ is finitely generated as a module over $\operatorname{HH}^{*}(\Lambda)$, and this shows that $\operatorname{Ext}_{\Lambda}^{*}(\Lambda / \mathfrak{r}, \Lambda / \mathfrak{r})$ is a finitely generated $\mathrm{HH}^{*}(\Lambda)$-module. The following result is therefore an application of Corollary 4.3.

Theorem 4.4. Let $k$ be a field, let $\Lambda$ be a symmetric periodic $k$-algebra with Jacobson radical $\mathfrak{r}$, and suppose that $\Lambda / \mathfrak{r} \otimes_{k} \Lambda / \mathfrak{r}$ is semisimple. Then for all modules $M, N \in$ $\bmod \Lambda$, the following are equivalent:

(i) $\operatorname{Ext}_{\Lambda}^{i}(M, N)=0$ for $i \gg 0$.

(ii) $\operatorname{Ext}_{\Lambda}^{i}(M, N)=0$ for $i>0$. 
(iii) $\operatorname{Ext}_{\Lambda}^{i}(N, M)=0$ for $i \gg 0$.

(iv) $\operatorname{Ext}_{\Lambda}^{i}(N, M)=0$ for $i>0$.

We finish this paper with an example where we look at self-injective Nakayama algebras. As we have seen, these algebras are periodic and therefore have finitely generated cohomology. However, the example shows that unless the algebra is symmetric, symmetry does not necessarily hold in the vanishing of cohomology.

Example 4.5. Let $\Gamma$ be the circular quiver

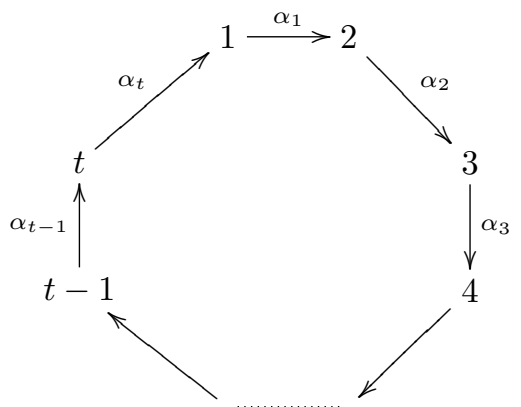

where $t \geqslant 2$ is an integer. Let $k$ be a field, denote by $k \Gamma$ the path algebra of $\Gamma$ over $k$, and let $J \subset k \Gamma$ be the ideal generated by the arrows. Fix an integer $n \geqslant 1$, let $\Lambda$ be the quotient algebra $k \Gamma / J^{n+1}$, and denote by $\mathfrak{r}$ the Jacobson radical of $\Lambda$. Then $\Lambda$ is a finite dimensional indecomposable self-injective Nakayama algebra, and $\operatorname{Ext}_{\Lambda}^{*}(\Lambda / \mathfrak{r}, \Lambda / \mathfrak{r})$ is a finitely generated $\mathrm{HH}^{*}(\Lambda)$-module (the ring structure of $\mathrm{HH}^{*}(\Lambda)$ was studied and determined in $[\mathbf{7}]$ and $[\mathbf{1 7}])$.

Write $n=q t+r$, where $0 \leqslant r<t$. Let $S_{i}$ be the simple module corresponding to the vertex $i$ and $P_{i}$ its projective cover. There is an exact sequence

$$
0 \rightarrow \Omega_{\Lambda}^{2}\left(S_{i}\right) \rightarrow P_{i+1(\bmod t)} \stackrel{\cdot \alpha_{i}}{\longrightarrow} P_{i} \rightarrow S_{i} \rightarrow 0
$$

and it is easy to see that $\Omega_{\Lambda}^{2}\left(S_{i}\right)$ is isomorphic to $S_{i+1+r(\bmod t)}$. Therefore the minimal projective resolution of $S_{i}$ is

$$
\cdots \rightarrow P_{i+3+2 r} \rightarrow P_{i+2+2 r} \rightarrow P_{i+2+r} \rightarrow P_{i+1+r} \rightarrow P_{i+1} \rightarrow P_{i} \rightarrow S_{i} \rightarrow 0,
$$

with $\Omega_{\Lambda}^{2 j}\left(S_{i}\right)=S_{i+j+j r}$ (all the indices are taken modulo $t$ ). A number of completely different situations may occur, depending on the values of the parameters $t$ and $r$. For example, if $r=0$, then we see that all the simple modules appear infinitely many times as even syzygies in the minimal projective resolution of any simple module. Therefore, in this case, if $S$ and $S^{\prime}$ are simple modules, then $\operatorname{Ext}_{\Lambda}^{n}\left(S, S^{\prime}\right)$ is nonzero for infinitely many $n$.

Note that when $r=0$, then $\Lambda$ is symmetric, and so by Theorem 4.4 symmetry holds in the vanishing of Ext. However, symmetry does not hold for all Nakayama algebras. For example, suppose that $t \geqslant 3$ and $r=t-1$. Then the exact sequences

$$
\begin{aligned}
& 0 \rightarrow S_{1} \rightarrow P_{2} \rightarrow P_{1} \rightarrow S_{1} \rightarrow 0 \\
& 0 \rightarrow S_{2} \rightarrow P_{3} \rightarrow P_{2} \rightarrow S_{2} \rightarrow 0
\end{aligned}
$$

are the first parts of the minimal projective resolutions of $S_{1}$ and $S_{2}$, and therefore 
$\operatorname{Ext}_{\Lambda}^{n}\left(S_{1}, S_{2}\right) \neq 0$ whenever $n$ is odd, whereas $\operatorname{Ext}_{\Lambda}^{n}\left(S_{2}, S_{1}\right)=0$ for all $n$. Thus in this situation there is no symmetry in the vanishing of Ext over $\Lambda$.

\section{Acknowledgements}

I would like to thank Steffen Oppermann and Idun Reiten for valuable comments on this paper.

\section{References}

[1] M.F. Atiyah and I.G. Macdonald, Introduction to commutative algebra, Addison-Wesley Publ. Co., Reading, Mass., 1969.

[2] M. Auslander and R.-O. Buckweitz, The homological theory of maximal CohenMacaulay approximations, in Colloque en l'honneur de Pierre Samuel (Orsay, 1987), Mém. Soc. Math. France 38 (1989), 5-37.

[3] M. Auslander and I. Reiten, Cohen-Macaulay and Gorenstein Artin algebras, in Representation theory of finite groups and finite-dimensional algebras (Bielefeld, 1991), 221-245, Progr. Math. 95, Birkhäuser, Basel, 1991.

[4] L.L. Avramov, Modules of finite virtual projective dimension, Invent. Math. 96 (1989), no. 1, 71-101.

[5] L.L. Avramov and R.-O. Buchweitz, Support varieties and cohomology over complete intersection, Invent. Math. 142 (2000), no. 2, 285-318.

[6] L.L. Avramov and S.B. Iyengar, Modules with prescribed cohomological support, Illinois J. Math. 51 (2007), no. 1, 1-20.

[7] M.J. Bardzell, A.C. Locateli and E.N. Marcosa, On the Hochschild cohomology of truncated cycle algebras, Comm. Alg. 28 (2000), no. 3, 1615-1639.

[8] D.J. Benson, Representations and cohomology. II, Cambridge Stud. Adv. Math. 31, Cambridge University Press, Cambridge, 1991.

[9] D.J. Benson, S.B. Iyengar and H. Krause, Local cohomology and support for triangulated categories, Ann. Sci. Éc. Norm. Supér. (4) 41 (2008), no. 4, 573619.

[10] P.A. Bergh, On complexes of finite complete intersection dimension, Homology, Homotopy Appl. 11 (2009), no. 2, 49-54.

[11] P.A. Bergh, S.B. Iyengar, H. Krause and S. Oppermann, Dimensions of triangulated categories via Koszul objects, Math. Z. 265 (2010), no. 4, 849-864.

[12] A.I. Bondal and M.M. Kapranov, Representable functors, Serre functors, and reconstructions (Russian), Izv. Akad. Nauk SSSR Ser. Mat. 53 (1989), no. 6, 1183-1205, 1337; translation in Math. USSR-Izv. 35 (1990), no. 3, 519-541.

[13] R.-O. Buchweitz, Maximal Cohen-Macaulay modules and Tate-cohomology over Gorenstein rings, unpublished manuscript (1987), 155 pp.

[14] R.-O. Buchweitz and H. Flenner, Global Hochschild (co-)homology of singular spaces, Adv. Math. 217 (2008), no. 1, 205-242.

[15] L.W. Christensen and H. Holm, Algebras that satisfy Auslander's condition on vanishing of cohomology, Math. Z. 265 (2010), no. 1, 21-40. 
[16] K. Erdmann, M. Holloway, R. Taillefer, N. Snashall and O. Solberg, Support varieties for selfinjective algebras, $K$-theory 33 (2004), no. 1, 67-87.

[17] K. Erdmann and T. Holm, Twisted bimodules and Hochschild cohomology for self-injective algebras of class $A_{n}$, Forum Math. 11 (1999), no. 2, 177-201.

[18] K. Erdmann, T. Holm and N. Snashall, Twisted bimodules and Hochschild cohomology for self-injective algebras of class $A_{n}$, II, Algebr. Represent. Theory 5 (2002), no. 5, 457-482.

[19] K. Erdmann and A. Skowroński, Periodic algebras, in Trends in representation theory of algebras and related topics, EMS Series of Congress Reports, European Mathematical Society, Zürich, 2008.

[20] K. Erdmann and N. Snashall, Preprojective algebras of Dynkin type, periodicity and the second Hochschild cohomology, in Algebras and modules II (Geiranger, 1996), 183-193, CMS Conf. Proc. 24, Amer. Math. Soc., Providence, RI, 1998.

[21] L. Evens, The cohomology ring of a finite group, Trans. Amer. Math. Soc. 101 (1961), 224-239.

[22] E. Green, N. Snashall and Ø. Solberg, The Hochschild cohomology ring of a selfinjective algebra of finite representation type, Proc. Amer. Math. Soc. 131 (2003), no. 11, 3387-3393.

[23] T.H. Gulliksen, On the deviations of a local ring, Math. Scand. 47 (1980), no. $1,5-20$.

[24] D. Happel, Triangulated categories in the representation theory of finitedimensional algebras, London Mathematical Society Lecture Note Series 119, Cambridge University Press, Cambridge, 1988.

[25] D. Happel, On Gorenstein rings, in Representation theory of finite groups and finite-dimensional algebras (Bielefeld, 1991), 389-404, Progr. Math. 95, Birkhäuser, 1991.

[26] D. Jorgensen, Vanishing of (co)homology over commutative rings, Comm. Algebra 29 (2001), no. 5, 1883-1898.

[27] B. Keller, Calabi-Yau triangulated categories, in Trends in representation theory of algebras and related topics, EMS Series of Congress Reports, European Mathematical Society, Zürich, 2008.

[28] I. Mori, Symmetry in the vanishing of Ext over stably symmetric algebras, $J$. Algebra 310 (2007), no. 2, 708-729.

[29] J. Rickard, Derived categories and stable equivalence, J. Pure Appl. Algebra 61 (1989), no. 3, 303-317.

[30] N. Snashall and Ø. Solberg, Support varieties and Hochschild cohomology rings, Proc. London Math. Soc. (3) 88 (2004), no. 3, 705-732.

[31] Ø. Solberg, Support varieties for modules and complexes, in Trends in representation theory of algebras and related topics, 239-270, Contemp. Math. 406, Amer. Math. Soc., Providence, RI, 2006.

[32] B.B. Venkov, Cohomology algebras for some classifying spaces (Russian), Dokl. Akad. Nauk SSSR 127 (1959), 943-944. 
[33] B.B. Venkov, Characteristic classes for finite groups (Russian), Dokl. Akad. Nauk SSSR 137 (1961), 1274-1277.

[34] J.L. Verdier, Des catégories dérivées des catégories abéliennes, Astérisque 239 (1996).

Petter Andreas Bergh bergh@math.ntnu.no

Institutt for matematiske fag, NTNU, N-7491 Trondheim, Norway 